\title{
Selective Inhibition of FLICE-like Inhibitory Protein (FLIP) Expression With Small Interfering RNA Oligonucleotides (siRNAs) Is Sufficient to Sensitize Tumor Cells for TRAIL-Induced Apoptosis
}

\author{
Daniela Siegmund, ${ }^{1}$ Philipp Hadwiger, ${ }^{2}$ Klaus Pfizenmaier, ${ }^{1}$ Hans-Peter Vornlocher, ${ }^{2}$ \\ and Harald Wajant ${ }^{1}$ \\ ${ }^{1}$ Institute of Cell Biology and Immunology, University of Stuttgart, Stuttgart, Germany \\ ${ }^{2}$ Ribopharma AG, Kulmbach, Germany \\ Accepted September 5, 2002
}

\begin{abstract}
Background: Most tumors express death receptors and their activation represents a potential selective approach in cancer treatment. The most promising candidate for tumor selective death receptor-activation is tumor necrosis factor-related apoptosis-inducing ligand (TRAIL)/Apo2L, which activates the death receptors TRAIL-R 1 and TRAIL$\mathrm{R} 2$, and induces apoptosis preferentially in tumor cells but not in normal tissues. However, many cancer cells are not or only moderately sensitive towards TRAIL and require cotreatment with irradiation or chemotherapy to yield a therapeutically reasonable apoptotic response. Because chemotherapy can have a broad range of unwanted side effects, more specific means for sensitizing tumor cells for TRAIL are desirable. The expression of the cellular FLICE-like inhibitory protein (cFLIP) is regarded as a major cause of TRAIL resistance. We therefore analyzed the usefulness of targeting FLIP to sensitize tumor cells for TRAIL-induced apoptosis.

Materials and Methods: To selectively interfere with expression of cFLIP short double-stranded RNA
\end{abstract}

oligonucleotides (small interfering RNAs [siRNAs]) were introduced in the human cell lines SV80 and KB by electroporation. Effects of siRNA on FLIP expression were analyzed by Western blotting and RNase protection assay and correlated with TRAIL sensitivity upon stimulation with recombinant soluble TRAIL and TRAIL-RI- and TRAIL-R2-specific agonistic antibodies.

Results: FLIP expression can be inhibited by RNA interference using siRNAs, evident from reduced levels of FLIP-mRNA and FLIP protein. Inhibition of cFLIP expression sensitizes cells for apoptosis induction by TRAIL and other death ligands. In accordance with the presumed function of FLIP as an inhibitor of death receptor-induced caspase- 8 activation, down-regulation of FLIP by siRNAs enhanced TRAIL-induced caspase- 8 activation.

Conclusion: Inhibition of FLIP expression was sufficient to sensitize tumor cells for TRAIL-induced apoptosis. The combination of TRAIL and FLIP-targeting siRNA could therefore be a useful strategy to attack cancer cells, which are resistant to TRAIL alone.

\section{Introduction}

Tumor necrosis factor (TNF)-related apoptosis-inducing ligand (TRAIL/Apo2L) is a typical member of the TNF ligand family that activates the apoptotic program in a variety of malignant cells but shows only limited cytotoxicity on normal cells $(1,2)$. TRAIL has therefore gained much attention as a potential anticancer agent. In support of a tumorrestricted action of TRAIL, first preclinical studies with a soluble nonaggregated form of TRAIL in mice and nonhuman primates gave no indication of systemic side effects $(3,4)$. However, it is important to note that systemic cytotoxicity does occur when aggregated soluble derivatives of TRAIL are applied $(5,6)$.

Because TRAIL exerts its pro-apoptotic properties by interaction with two death domain-containing

Address correspondence and reprint requests to: Harald Wajant Institute of Cell Biology and Immunology, University of Stuttgart, Allmandring 31, 70569 Stuttgart, Germany. Phone: 49711685 7446; fax: 49711685 7484;

e-mail: harald.wajant@po.uni-stuttgart.de. members of the TNF receptor superfamily, TRAILR 1 and TRAIL-R2 $(1,2)$, the differential reactivity on normal tissues of aggregated and nonaggregated soluble TRAIL variants may be related to the differential activation of the TRAIL death receptors by membrane bound TRAIL and soluble TRAIL (7). In support of this reasoning, systemic toxicity of membrane TRAIL has been reported (8). Stimulation of TRAIL-R I or TRAIL-R2 results in Fas-associated death domaincontaining protein (FADD)-dependent recruitment of procaspase-8 and -10 (9-11). Proximity-induced processing of pro-caspase- 8 and -10 in the death-inducing signaling complex of TRAIL-R1 and TRAIL-R2 results in the release of processed (active) caspase-8 and -10 , and consequently in induction of apoptosis (9-12).

In addition, TRAIL interacts with three other members of the TNF receptor superfamily, namely TRAIL-R3, TRAIL-R4, and osteoprotegerin (OPG) that act as decoy receptors antagonizing the apoptotic capacity of TRAIL $(1,2)$. In many cases, however, lack of decoy receptor expression does not 
correlate with TRAIL sensitivity. Thus, other determinants must exist to regulate the sensitivity of cells toward TRAIL. In this regard, cellular FLICEinhibitory protein (CFLIP) has been identified as a major cellular inhibitor of apoptosis induced by TRAIL-R $1 / 2$ and other death receptors (13). cFLIP is similar in its structure to caspase- 8 and -10 , but lacks enzymatic activity. Although more than 10 splice variants of cFLIP have been found at the mRNA level, only two of them, FLIP-short (FLIP-S) and FLIP-long (FLIP-L), have been extensively studied (13). FLIP-L consists of two amino-terminal death effector domains (DED) and a caspase homology domain without catalytic activity. In its overall architecture, FLIP-L resembles caspase-8 and -10, whereas FLIP-S solely comprises the two amino-terminal DEDs of FLIP-L (13). Both FLIP-S and FLIP$\mathrm{L}$ interact with FADD and caspase- 8 and are consequently recruited into the death-inducing signaling complex of TRAIL-R 1 or TRAIL-2. Here, these molecules interfere with the processing of procaspase- 8 and - 10 (13-15).

Many tumor cells are resistant or only moderately sensitive against TRAIL-mediated apoptosis, but can be significantly sensitized by chemotherapeutic drugs or $\gamma$-irradiation. In good agreement with a major role of FLIP in the regulation of TRAIL-induced apoptosis, it has been shown in several studies that chemotherapy inhibits FLIP expression. In particular the NF- $\kappa$ B $(16,17)$, Akt $(18-20)$, PKCs $(21)$, and MAPK $(22-24)$ pathways have been implicated in regulation of FLIP expression and pharmacologic interference with these pathways can sensitize for TRAIL-induced apoptosis. Here we show that highly selective inhibition of cFLIP expression by RNA interference using short, sequence-specific double-stranded RNA oligonucleotides (small interfering RNAs [siRNAs]) is sufficient to sensitize tumor cell lines for TRAIL-mediated apoptosis. Thus, our study indicates that it is feasible to target a single molecule by siRNAs to enhance the apoptotic effects of TRAIL. The implementation of this selective RNA targeting approach in TRAIL-based therapies opens the possibility to circumvent broad effects common to chemotherapeutic drugs.

\section{Material and Methods \\ Materials}

The human SV40-transformed fibroblast cell line SV80 was originally described by Todaro et al. (25) and was a kind gift of $\mathrm{H}$. Engelmann (University of Munich, Munich, Germany). The human epidermal cell line KB was obtained from the American Type Culture Collection (Rockville, MD, USA). Cells were maintained in Click's RPMI medium (Biochrom, Berlin, Germany) supplemented with 5\% fetal calf serum (FCS). KB-FLIP-L-GFP and KB-FLIP-S-GFP cells were derived from parental KB cells by stable transfection with expression plasmids encoding green fluorescent protein (GFP) fusion proteins of the long (FLIP-L) and short (FLIP-S) splice form of FLIP. To obtain polyclonal populations of FLIP-LGFP and FLIP-S-GFP expressing KB cells, about 100 primary clones were pooled, expanded, and sorted twice for expression of the GFP fusion protein using a FACStarPlus cell sorter (Becton Dickinson, Heidelberg, Germany). TRAIL-M2 complex was generated by mixing the respective concentration of Flagtagged TRAIL with the anti-Flag monoclonal antibody M2 (Sigma-Aldrich, Deisenhofen, Germany) to a final concentration of $1 \mu \mathrm{g} / \mathrm{ml}$ of antibody. After 15 min incubation at room temperature, the formed TRAIL-M2-complex was transferred to the cells. Flag-tagged TRAIL was generously supplied by P. Schneider and J. Tschopp (University of Lausanne, Lausanne, Switzerland). Agonistic TRAIL-R I and TRAIL-R2 specific rabbit antisera were described elsewhere (26). The caspase-8-specific mAb was a kind gift of Klaus Schulze-Osthoff (University of Düsseldorf, Düsseldorf, Germany). Goat antimouse-IgG, NBT and BCIP were from Sigma (Deisenhofen, Germany).

\section{Production of Small Interfering Oligoribonucleotides}

Small interfering oligoribonucleotides (siRNAs) specific for nucleotides 472-492 (siRNA-F1) and 908-928 (siRNA-F2) of FLIP-L (ac.-no.: U97074), nucleotides 843-863 (siRNA-GFP) of pEGFP-C1 (ac.-no.: U55673), and nucleotides 2610-2630 (siRNA-neo) of the neomycin-phosphotransferase gene of pEGFP-Cl (ac.-no.: U55673) were synthesized on an Expedite 8909 Synthesizer (Applied Biosystems, Germany) at $1 \mu$ mole scale on solid phase employing Expedite RNA phosphoramidites (Proligo, Germany) (27). Deprotection and gel purification of oligoribonucleotides were carried out according to procedures described elsewhere (28). siRNAs were generated by mixing equimolar amounts of sense and antisense strands in annealing buffer ( $20 \mathrm{mM}$ sodium phosphate, pH 6.8; $100 \mathrm{mM}$ sodium chloride), heating in a water bath at $85-90{ }^{\circ} \mathrm{C}$ for $3 \mathrm{~min}$ and cooling to room temperature over a period of 3-4 hours. SiRNAs were stored in aliquots at $-20^{\circ} \mathrm{C}$.

\section{Electroporation}

For transfection cells $\left(2-10 \times 10^{6}\right.$ cells $\left./ \mathrm{ml}\right)$ were electroporated with $150 \mathrm{nM}$ of the indicated siRNA by electroporation ( $4 \mathrm{~mm}$ cuvette; $250 \mathrm{~V}, 1800 \mu \mathrm{F}$, maximal resistance) in medium with $5 \%$ FCS using an Easyject Plus (PeqLab, Germany). In some experiments cells were electroporated a second time after 1 day of recovery to increase the fraction of successfully transfected cells.

\section{FACS Analysis}

GFP and the GFP fusion proteins FLIP-S-GFP and FLIP-L-GFP were detected with a FACStar ${ }^{\text {Plus }}$ (Becton Dickinson). FACS analysis of GFP was used to control electroporation efficiency of cotransfected siRNAs. Alterations in the expression level of FLIP-S-GFP 
and FLIP-L-GFP expressing KB transfectants were determined by FACS analysis as follows: A gate was defined with parental KB cells, where fewer than $2 \%$ of the cells were indicated as positive cells. Using the same settings, relative expression of the GFP fusion proteins of FLIP-L and FLIP-S in KB-FLIP-L-GFP and KB-FLIP-S-GFP cells was determined by calculating the product of percentage of positive cells and their mean fluorescence intensity. The product of percentage of positive cells and the corresponding mean fluorescence intensity of mock electroporated cells was defined as $100 \%$ in each case.

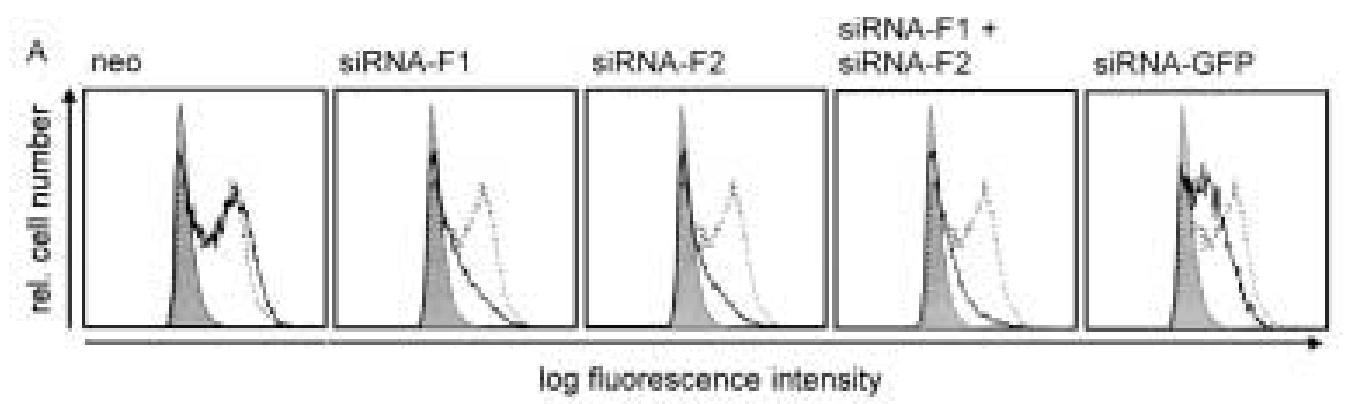

B

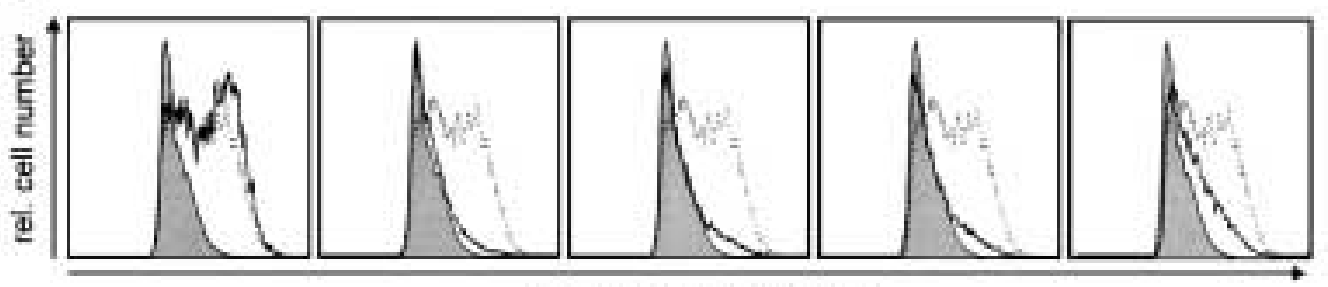

$\log$ fluorescence intensity

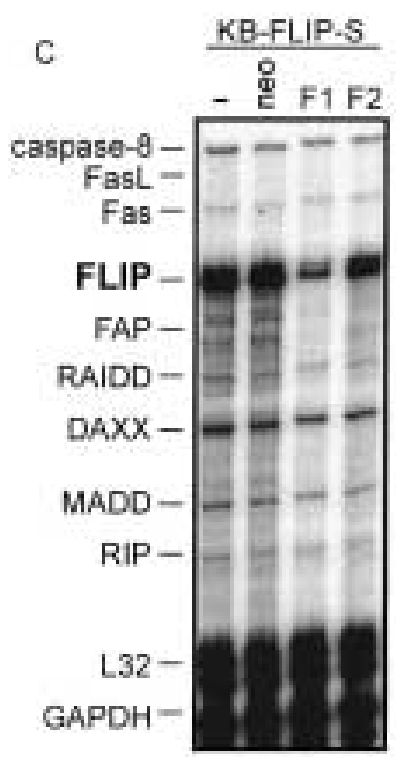

L-32 noimalized

FLIP expression

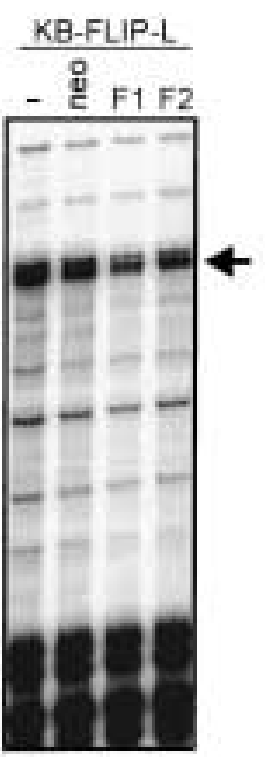

$+0.90 .50 .7$

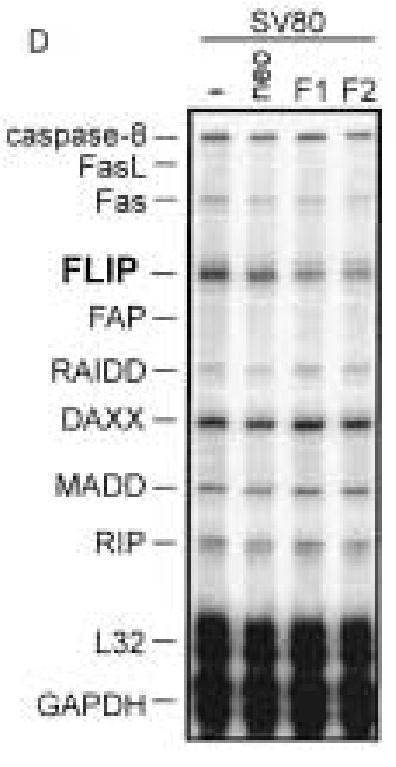

$\begin{array}{lllll}1 & 0.8 & 0.4 & 0.4\end{array}$

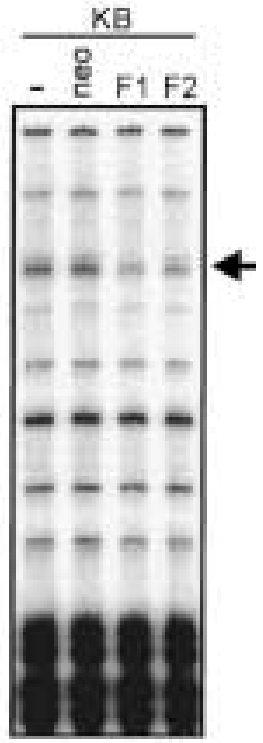

$+10.50 .5$

Fig. 1. FLIP-specific short double-stranded RNA oligonucleotides inhibit FLIP expression. (A and B) KB cells stably transfected with fusion proteins of FLIP-L (A) and FLIP-S (B) carboxy-terminally tagged with EGFP (KB-FLIP-L-GFP; KB-FLIP-S-GFP) were transfected twice on 2 successive days by electroporation with the indicated siRNAs. One day after the second round of electroporation expression of the GFP, chimeras were analyzed by FACS (solid line) and compared with parental KB cells (grey), untreated KB-FLIP-L-GFP (dotted lines in A) and KB-FLIP-S-GFP cells (dotted lines in B). (C and D) KB-FLIP-L-GFP (C), KB-FLIP-S-GFP (C), KB (D), and SV80 cells (D) were electroporated twice with the indicated siRNAs as described and after l day of recovery total RNAs were isolated. RPA analyses were performed to detect transcripts of the indicated genes using the Multi-Probe template set hApo-3b (PharMingen). Protected transcripts were separated by denaturing polyacrylamide gel electrophoresis (5\%) and visualized on a PhosphorImager. Protected FLIP transcripts were normalized with respect to RNA loads using the L32 internal expression control. 


\section{RNAse Protection Assay}

Total RNA was isolated with the RNA INSTAPURE kit (Eurogentech, Belgium) according to the manufacturer's recommendations. The presence of transcripts of the indicated genes, including FLIP and the internal controls L32 and GAPDH, were analyzed using the Multi-Probe template set hApo-3b (PharMingen, Hamburg, Germany). Probe synthesis, hybridization, and RNase treatment were performed with the RiboQuant Multi-Probe RNase Protection Assay System (PharMingen) according to the manufacturer's recommendations. After RNase treatment the protected transcripts were resolved by electrophoresis on a denaturing polyacrylamide gel $(5 \%)$ and analyzed on a PhosphorImager with the ImageQuant software (Molecular Dynamics, Sunnyvale, CA, USA).

\section{Western Blotting}

To prepare cell lysates RIPA buffer supplemented with 0.1 vol of a protease inhibitor cocktail stock solution (Roche, Mannheim, Germany) was used. After centrifugation at $10000 \times \mathrm{g}$ for $10 \mathrm{~min}$ to remove insoluble cell debris, protein concentrations were determined by the Bradford assay. Protein $(100 \mu \mathrm{g})$ were boiled in sample buffer $(5 \mathrm{~min})$ and

Table 1. siRNA-mediated reduction of FLIP-L-GFP expression in KB-FLIP-L-GFP cells

\begin{tabular}{|c|c|c|c|c|c|c|}
\hline siRNA & $\mathbf{n M}$ & hr & Positive Cells (\%) & Mean Intensity & Relative Expression & Reduction (\%) \\
\hline & 0 & 24 & 48 & 30 & 1440 & 0 \\
\hline siRNA-Fl & 75 & 24 & 24 & 26 & 624 & 57 \\
\hline siRNA-Fl & 150 & 24 & 14 & 23 & 322 & 78 \\
\hline siRNA-Fl & 300 & 24 & 9 & 22 & 198 & 87 \\
\hline siRNA-F2 & 75 & 24 & 11 & 24 & 264 & 82 \\
\hline siRNA-F2 & 150 & 24 & 15 & 23 & 345 & 75 \\
\hline siRNA-F2 & 300 & 24 & 7 & 25 & 175 & 88 \\
\hline siRNA-neo & 75 & 24 & 49 & 27 & 1323 & 8 \\
\hline siRNA-neo & 150 & 24 & 54 & 27 & 1458 & -1 \\
\hline \multirow[t]{2}{*}{ siRNA-neo } & 300 & 24 & 52 & 28 & 1456 & -1 \\
\hline & & 48 & 52 & 25 & 1300 & 0 \\
\hline siRNA-Fl & 75 & 48 & 33 & 23 & 759 & 42 \\
\hline siRNA-Fl & 150 & 48 & 23 & 21 & 483 & 63 \\
\hline siRNA-F1 & 300 & 48 & 3 & 17 & 51 & 97 \\
\hline SiRNA-F2 & 75 & 48 & 15 & 21 & 315 & 76 \\
\hline siRNA-F2 & 150 & 48 & 10 & 21 & 210 & 84 \\
\hline SiRNA-F2 & 300 & 48 & 9 & 21 & 189 & 86 \\
\hline siRNA-neo & 75 & 48 & 53 & 26 & 1378 & -6 \\
\hline siRNA-neo & 150 & 48 & 51 & 24 & 1224 & 6 \\
\hline \multirow[t]{2}{*}{ siRNA-neo } & 300 & 48 & 46 & 24 & 1104 & 16 \\
\hline & & 96 & 40 & 24 & 960 & 0 \\
\hline siRNA-Fl & 75 & 96 & 33 & 22 & 726 & 25 \\
\hline siRNA-Fl & 150 & 96 & 26 & 21 & 546 & 44 \\
\hline siRNA-Fl & 300 & 96 & 12 & 21 & 252 & 74 \\
\hline siRNA-F2 & 75 & 96 & 27 & 21 & 567 & 61 \\
\hline siRNA-F2 & 150 & 96 & 29 & 21 & 609 & 63 \\
\hline siRNA-F2 & 300 & 96 & 17 & 20 & 340 & 65 \\
\hline siRNA-neo & 75 & 96 & 45 & 24 & 1080 & -12 \\
\hline siRNA-neo & 150 & 96 & 49 & 26 & 1274 & -32 \\
\hline siRNA-neo & 300 & 96 & 44 & 25 & 1100 & -14 \\
\hline
\end{tabular}

Twenty-four, 48, and $96 \mathrm{hr}$, after two rounds of electroporation with the indicated concentration of siRNA-F1, siRNA-F2, and siRNAneo, cells were analyzed by FACS to determine relative FLIP-L-GFP expression. The product of percentage of positive cells and the corresponding mean fluorescence intensity of mock electroporated cells was defined as $100 \%$. 
resolved by sodium dodecyl sulfate-polyacrylamide gel electrophoresis (SDS-PAGE). After transfer to nitrocellulose membranes by electroblotting, nonspecific binding sites on the membranes were blocked by incubation in phosphate-buffered saline (PBS) containing $0.05 \%$ Tween-20 and $3 \%(\mathrm{w} / \mathrm{v})$ dry milk. Procaspase- 8 (p55/53) and the p18 and p41/43 fragments derived thereof by proteolytic processing were detected with a caspase-8-specific $\mathrm{mAb}$ and horseradish peroxidase-conjugated goat anti-mouse-IgG using NBT and BCIP as substrates.

\section{Cytotoxicity Assay}

After the second electroporation cells $\left(20 \times 10^{3}\right)$ were grown overnight in $100 \mu \mathrm{l}$ of culture medium in 96-well plates. The next day, cells were treated with the indicated concentrations of TRAIL, TRAILM2 complex, or TRAIL-R1- and TRAIL-R2-specific rabbit antisera. Cell viability was determined using crystal violet staining as described elsewhere (29).

\section{Results and Discussion}

FLIP-Specific Small Interfering Oligoribonucleotides Inhibit FLIP Expression

To inhibit FLIP expression two siRNAs of 21 nucleotides corresponding to nucleotides 472-492 (siRNA-F1) and 908-928 (siRNA-F2) of FLIP-L were generated and initially analyzed for their capability to reduce expression of a fusion protein of FLIP-L and GFP (FLIP-L-GFP). Transient cotransfection of siRNA-F1 and siRNA-F2 along with an expression vector encoding FLIP-L-GFP led to a 50-80\% reduced FLIP-L-GFP expression as shown by FACS analyses. In contrast, a control siRNA of similar structure, specific for the neomycin gene (siRNA-neo), showed no effects (data not shown).
Similarly, in KB cells stably expressing FLIP-L-GFP, transient introduction of siRNA-F1 and siRNA-F2 by two rounds of electroporation on consecutive days reduced FLIP-L-GFP expression by up to $90 \%$, whereas various control siRNAs including siRNAneo showed no significant effect (Fig. 1A, Table 1). siRNA-F1 and siRNA-F2 showed no effect on GFP expression (data not shown). The inhibitory effect of FLIP-specific siRNAs was presumably even higher because the transfection efficiency of $\mathrm{KB}$ cells is between 60 and $80 \%$ in a single round of electroporation (data not shown). Interference with FLIP-LGFP expression using a GFP-specific siRNA also resulted in a reduction of FLIP-L-GFP expression, but with a lower efficiency as compared to FLIP-specific siRNAs (Fig. 1A). siRNA-F2 inhibited expression of FLIP-S-GFP with comparable efficiency as FLIP-L-GFP expression (Fig. 1B, Table 2), although the siRNA-F2 target sequence is not part of the coding region of FLIP-S. Thus, the targeted exon could be part of the 3' untranslated region of the spliced mature mRNA of FLIP-S, but further studies are required to clarify this. In accordance with the concept that RNA interference targets mRNA, we found in RNase protection assays (RPA) of FLIP-L-GFP and FLIP-S-GFP-expressing KB cells a significant reduction in FLIP mRNA after electroporation with siRNA-F1 and siRNA-F2 (Fig. 1C). More importantly, in $\mathrm{KB}$ and SV80 cells, both FLIP-specific siRNAs significantly reduced endogenous FLIP-mRNA (Fig. 1D).

Inhibition of FLIP Expression Is Sufficient to Sensitize Cells for TRAIL-Induced Apoptosis

To analyze the effect of reduced expression levels of FLIP on TRAIL-induced apoptosis, KB and SV80 cells were analyzed. To optimize the overall

Table 2. siRNA-mediated reduction of FLIP-S-GFP expression in KB-FLIP-S-GFP cells

\begin{tabular}{|c|c|c|c|c|c|}
\hline SiRNA & hr & Positive Cells (\%) & Mean Intensity & Relative Expression & Reduction (\%) \\
\hline- & 24 & 46 & 30 & 1380 & 0 \\
\hline siRNA-F1 & 24 & 9 & 20 & 180 & 87 \\
\hline siRNA-F2 & 24 & 15 & 28 & 420 & 70 \\
\hline $\mathrm{F} 1+\mathrm{F} 2$ & 24 & 16 & 27 & 432 & 69 \\
\hline siRNA-neo & 24 & 48 & 30 & 1440 & -4 \\
\hline- & 48 & 53 & 37 & 1961 & 0 \\
\hline siRNA-F1 & 48 & 29 & 22 & 638 & 68 \\
\hline siRNA-F2 & 48 & 23 & 27 & 621 & 68 \\
\hline $\mathrm{F} 1+\mathrm{F} 2$ & 48 & 27 & 27 & 729 & 63 \\
\hline siRNA-neo & 48 & 54 & 37 & 1998 & -2 \\
\hline
\end{tabular}

Twenty-four and $48 \mathrm{hr}$, after two rounds of electroporation with the indicated siRNAs (150 nM), cells were analyzed by FACS to determine relative FLIP-S-GFP expression. The product of percentage of positive cells and the corresponding mean fluorescence intensity of mock electroporated cells was defined as $100 \%$. 

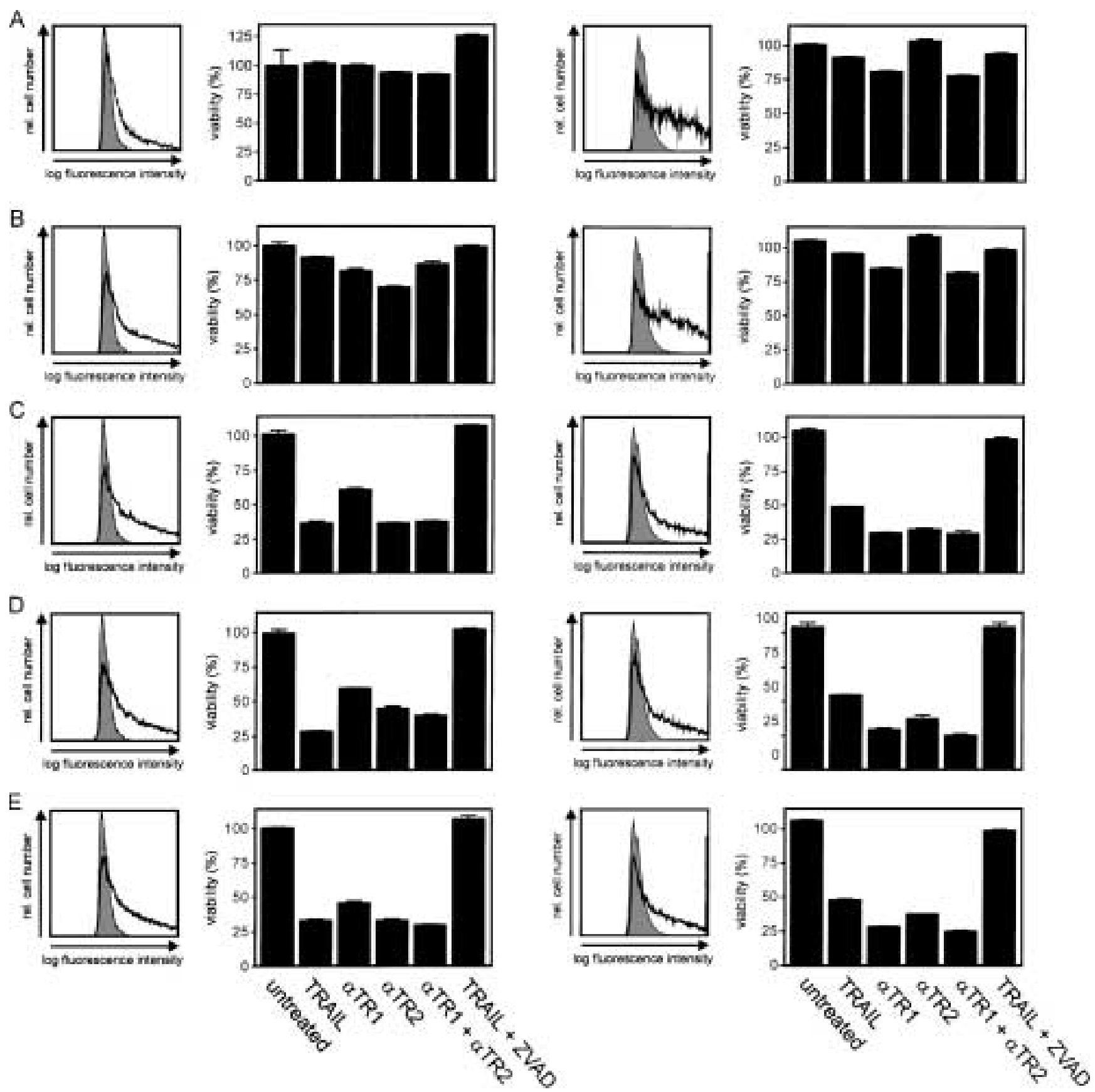

\section{SV80 cells}

\section{KB cells}

Fig. 2. FLIP-specific siRNAs sensitize tumor cells for TRAIL-induced apoptosis. SV80 and $\mathrm{KB}$ cells $\left(10^{7} \mathrm{cells} / \mathrm{ml}\right) \mathrm{were}$ transiently transfected twice by electroporation on 2 consecutive days without (A) or with 150 nM of siRNA-neo (B), siRNA-F1 (C), siRNA-F2 (D), or a mixture of $75 \mathrm{nM}$ each of siRNA-F1 and siRNA-F2 (E). In the first round of electroporation, a GFP expression plasmid was included in the electroporation mixture to verify transfection efficiencies. One day after the first electroporation, cells were harvested and analyzed for transfection efficiency by FACS analysis (solid line, first and third panel) or were electroporated a second time with the same siRNA as the first day. After the second electroporation cells were seeded in $100 \mu \mathrm{l}$ medium in 96-well plates. The next day, cells were treated for $9 \mathrm{hr}$ with Flag-tagged soluble TRAIL $(50 \mathrm{ng} / \mathrm{ml})$ cross-linked with anti-Flag mAb M2 $(1 \mu \mathrm{g} / \mathrm{ml})$ or agonistic TRAIL-R 1- and TRAIL-R2-specific rabbit antisera (1:500), or remained untreated. Where indicated, cells were challenged with TRAIL in the presence of the caspase inhibitor z-VAD-fmk $(20 \mu \mathrm{M})$. Finally, cell viability was determined using crystal violet staining (second and fourth panel). 
transfection efficiency of the siRNAs, we introduced siRNAs 2 consecutive days by electroporation. To allow quantification of the transfection efficiency, a GFP expression plasmid was cotransfected. After an additional recovery period of 1-2 days, cells were challenged with a soluble cross-linked TRAIL derivative that is able to stimulate both death domaincontaining TRAIL receptors, or with agonistic TRAIL-R 1- and TRAIL-R2- specific IgGs. As shown in Figure 2, the transfection efficiency of the GFP expression plasmid was not affected by the presence of siRNA in all cell lines investigated. siRNA-Fl and siRNA-F2 (Figs. 2C-2E), but not siRNA-neo or mock electroporation (Figs. 2A and 2B), significantly sensitized KB and SV80 cells for TRAIL-R Iand TRAIL-R2-mediated apoptosis. The broad range caspase inhibitor z-VAD-fmk inhibited TRAIL-induced cell death indicating the essential involvement of caspases (Figs. 2C-2E). KB cells were also sensitized for FasL- and TNF-induced apoptosis, but for the latter to a significantly lower extend as compared to TRAIL-induced apoptosis (data not shown). This is not unexpected; for TNFR l FLIP-independent NF-kB-mediated anti-apoptotic mechanisms exist (30), which may override the sensitizing effect of FLIP-specific RNA interference. In accordance with the function of FLIP, TRAIL-induced caspase- 8 activation was significantly increased in siRNA-F1 and siRNA-F2 treated cells (Fig. 3).

A possible complication that could severely limit the broad use of TRAIL in cancer therapy is that even trimeric soluble TRAIL may exert cytotoxic effects on normal tissue when a combined treatment with chemotherapeutic drugs is required. This possibility could gain great importance, because in many in vitro studies efficient killing of tumors cells by TRAIL critically depends on cotreatment with chemotherapeutic reagents (1). Indeed, it is not unlikely that some of the mechanisms responsible for protection of normal cells from TRAIL-induced apoptosis are targets of chemotherapeutic drugs. Because RNA interference is much more selective than chemotherapeutic drugs, siRNAs should efficiently sensitize for TRAIL-induced apoptosis with a considerably reduced risk of side effects compared to chemotherapy. A further application of a FLIP targeting strategy is envisaged in treatment modalities aiming at activation of p53 function, for example, by chemotherapy, thereby inducing, among several other p53 effects, the expression of Fas, its ligand FasL (31-33), and the TRAIL-death receptors $(1,2)$, thus rendering the affected tumor cells potentially responsive to undergo death receptor-induced apoptosis in an autocrine or paracrine manner. In this context, it is expected that down-regulation of FLIP would greatly enhance the sensitivity of tumor cells toward p53-dependent death receptormediated apoptosis.
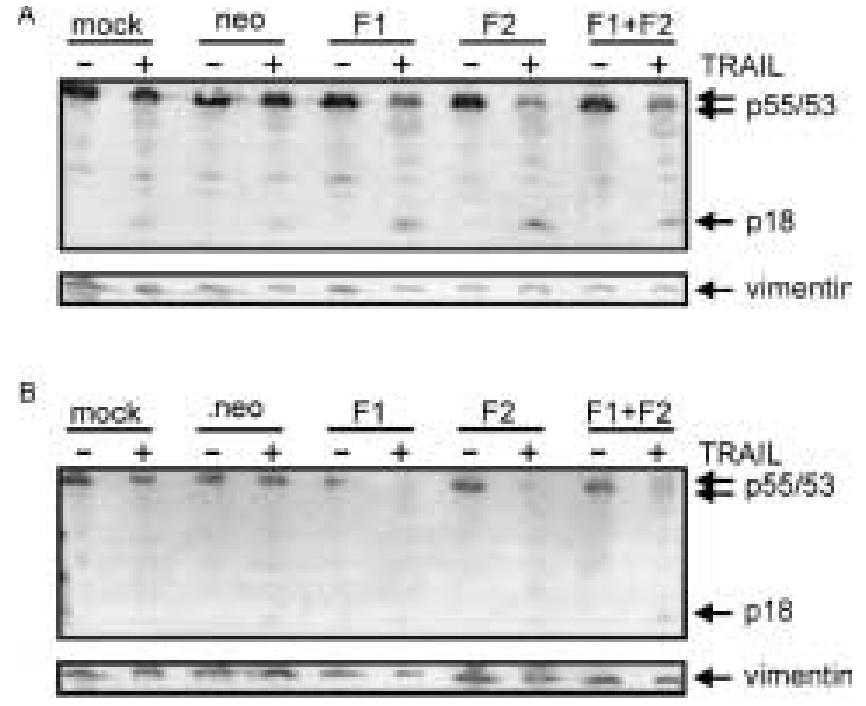

Fig. 3. FLIP-specific siRNAs enhance TRAIL-induced processing of caspase-8. SV80 (A) and KB cells (B) $\left(10^{7}\right.$ cells $\left./ \mathrm{ml}\right)$ were transiently transfected with the indicated siRNAs (150 nM) as described in Figure 2. After the second electroporation, cells were seeded in $2 \mathrm{ml}$ of medium in 6-well plates. The next, day cells were challenged for $4 \mathrm{hr}$ with anti-Flag $(1 \mu \mathrm{g} / \mathrm{ml})$ cross-linked TRAIL (100 ng/ml). Finally, detached and attached cells were harvested, lysed together in RIPA buffer supplemented with 0.1 vol of a protease inhibitor cocktail stock solution (Roche), and analyzed for procaspase- 8 processing by Western blotting using a caspase-8-specific monocolonal antibody $(\mathrm{mAb})$ recognizing the $\mathrm{p} 55 / 53$ procaspase- 8 isoforms and the processed p18 subunit. Western blotting with a vimentin-specific mAb served as control for protein loads.

\section{Acknowledgments}

We thank P. Schneider and J. Tschopp (University of Lausanne, Switzerland) for kindly providing Flagtagged TRAIL. We also thank Klaus Schulze-Osthoff (University of Düsseldorf, Germany) for kindly providing the mAb against caspase-8. This work was supported by Deutsche Forschungsgemeinschaft Grant Wa 1025/3-1 and Sonderforschungsbereich 495 project A5 and Deutsche Krebshilfe Grant 101751-Wa 3.

\section{References}

1. Srivastava RK. (2001) TRAIL/Apo-2L: mechanisms and clinical applications in cancer. Neoplasia 3: 535-546.

2. Nagane M, Huang HJ, Cavenee WK. (2001) The potential of TRAIL for cancer chemotherapy. Apoptosis 6: 191-197.

3. Ashkenazi A, Pai RC, Fong S, et al. (1999) Safety and antitumor activity of recombinant soluble Apo2 ligand. J. Clin. Invest. 104: 155-162.

4. Walczak H, Miller RE, Ariail K, et al. (1999) Tumoricidal activity of tumor necrosis factor-related apoptosis-inducing ligand in vivo. Nat. Med. 5: 157-163.

5. Jo M, Kim TH, Seol DW, et al. (2000) Apoptosis induced in normal human hepatocytes by tumor necrosis factor-related apoptosis-inducing ligand. Nat. Med. 6: 564-567.

6. Lawrence D, Shahrokh Z, Marsters S, et al. (2001) Differential hepatocyte toxicity of recombinant Apo2L/TRAIL versions. Nat. Med. 7: 383-385.

7. Wajant H, Moosmayer D, Wuest T, et al. (2001) Differential activation of TRAIL-R 1 and -2 by soluble and membrane 
TRAIL allows selective surface antigen-directed activation of TRAIL-R2 by a soluble TRAIL derivative. Oncogene 20: 4101-4106.

8. Ichikawa K, Liu W, Zhao L, et al. (2001) Tumoricidal activity of a novel anti-human DR5 monoclonal antibody without hepatocyte cytotoxicity. Nat. Med. 7: 954-960.

9. Bodmer JL, Holler N, Reynard S, et al. (2000) TRAIL receptor-2 signals apoptosis through FADD and caspase-8. Nat. Cell Biol. 2: 241-243.

10. Sprick MR, Weigand MA, Rieser E, et al. (2000) FADD/MORTI and caspase- 8 are recruited to TRAIL receptors 1 and 2 and are essential for apoptosis mediated by TRAIL receptor 2. Immunity 12: 599-609.

11. Kischkel FC, Lawrence DA, Chuntharapai A, Schow P, Kim KJ, Ashkenazi A. (2000) Apo2L/TRAIL-dependent recruitment of endogenous FADD and caspase- 8 to death receptors 4 and 5. Immunity 12: 611-620.

12. Kuang AA, Diehl G, Zhang J, Winoto A. (2000) FADD is required for DR4- and DR5-mediated apoptosis: lack of trailinduced apoptosis in FADD-deficient mouse embryonic fibroblasts. J. Biol. Chem. 275: 25065-25068.

13. Krueger A, Baumann S, Krammer PH, Kirchhoff S. (2001) FLICE-inhibitory proteins: regulators of death receptor-mediated apoptosis. Mol. Cell Biol. 21: 8247-8254.

14. Kischkel FC, Lawrence DA, Tinel A, et al. (2001) Death receptor recruitment of endogenous caspase-10 and apoptosis initiation in the absence of caspase-8. J. Biol. Chem. 276: 46639-46646.

15. Wang J, Chun HJ, Wong W, Spencer DM, Lenardo MJ. (2001) Caspase-10 is an initiator caspase in death receptor signaling. Proc. Natl. Acad. Sci. U.S.A. 98: 13884-13888.

16. Kreuz S, Siegmund D, Scheurich P, Wajant H. (2001) NFkappaB inducers upregulate cFLIP, a cycloheximide-sensitive inhibitor of death receptor signaling. Mol. Cell Biol. 21: 3964-3973.

17. Micheau O, Lens S, Gaide O, Alevizopoulos K, Tschopp J. (2001) NF-kappaB signals induce the expression of c-FLIP. Mol. Cell Biol. 21: 5299-5305.

18. Nam SY, Amoscato AA, Lee YJ. (2002) Low glucoseenhanced TRAIL cytotoxicity is mediated through the ceramide-Akt-FLIP pathway. Oncogene 21: 337-346.

19. Suhara T, Mano T, Oliveira BE, Walsh K. (2001) Phosphatidylinositol 3-kinase/Akt signaling controls endothelial cell sensitivity to Fas-mediated apoptosis via regulation of FLICE-inhibitory protein (FLIP). Circ. Res. 89: 13-19.
20. Panka DJ, Mano T, Suhara T, Walsh K, Mier JW. (2001) Phosphatidylinositol 3-kinase/Akt activity regulates c-FLIP expression in tumor cells. J. Biol. Chem. 276: 6893-6896.

21. Gomez-Angelats M, Cidlowski JA. (2001) Protein kinase C regulates FADD recruitment and death-inducing signaling complex formation in Fas/CD95-induced apoptosis. J. Biol. Chem. 276: 44944-44952.

22. Tran SE, Holmstrom TH, Ahonen M, Kahari VM, Eriksson JE. (2001) MAPK/ERK overrides the apoptotic signaling from Fas, TNF, and TRAIL receptors. J. Biol. Chem. 276: 16484-16490.

23. Wang W, Prince C, Mou Y, Pollman M (2002). Notch3 signaling in vascular smooth muscle cells induces c-FLIP expression via ERK/MAPK activation: resistance to FasL-induced apoptosis. J. Biol. Chem. 277: 21723-21729.

24. Aoudjit F, Vuori K. (2001) Matrix attachment regulates Fasinduced apoptosis in endothelial cells: a role for c-flip and implications for anoikis. J. Cell Biol. 152: 633-643.

25. Todaro JG, Green H, Swift MR. (1966) Science 153: 1252-1254.

26. Muhlenbeck F, Schneider P, Bodmer JL, et al. (2000). J. Biol. Chem. 275: 32208-32213.

27. Vargeese C, Carter J, Yegge J, et al. (1998) Efficient activation of nucleoside phosphoramidites with 4,5-dicyanoimidazole during oligonucleotide synthesis. Nucleic Acids Res. 26: 1046-1050.

28. Wincott F, DiRenzo A, Shaffer C, et al. (1995) Synthesis, deprotection, analysis and purification of RNA and ribozymes. Nucleic Acids Res. 23: 2677-2684.

29. Weiss T, Grell M, Hessabi B, et al. (1997) Enhancement of TNF receptor $\mathrm{p} 60$-mediated cytotoxicity by TNF receptor $\mathrm{p} 80$ : requirement of the TNF receptor-associated factor- 2 binding site. J. Immunol. 158: 2398-2404.

30. Wajant H, Scheurich P. (2001) Tumor necrosis factor receptorassociated factor (TRAF) 2 and its role in TNF signaling. Int. J. Biochem. Cell Biol. 33: 19-32.

31. Owen-Schaub LB, Zhang W, Cusack JC, et al. (1995) Wildtype human p53 and a temperature-sensitive mutant induce Fas/APO-1 expression. Mol. Cell Biol. 15: 3032-3040.

32. Muller M, Strand S, Hug H, et al. (1997) Drug-induced apoptosis in hepatoma cells is mediated by the CD95 (APO-1/Fas) receptor/ligand system and involves activation of wild-type p53. J. Clin. Invest. 99: 403-413.

33. Muller M, Wilder S, Bannasch D, et al. (1998) p53 activates the CD95 (APO-1/Fas) gene in response to DANN damage by anticancer drugs. J. Exp. Med. 188: 2033-2045. 